\title{
EEG signal-based movement control for mobile robots
}

\author{
Yumlembam Rahul* and Rupam Kumar Sharma \\ Department of Computer Science and Engineering \& Information Technology, School of Technology, Assam Don Bosco University, \\ Azara 781017 , India
}

\begin{abstract}
Although wheelchair with joystick control is available, people whose hands are paralysed cannot use the joystick and need other forms of assistance to move. This article presents the design and analysis of a mobile prototype robot control using a single-electrode commercial electroencephalogram (EEG) headset. We examine the possibility of detecting P300 and blink signal for use as an input to control a prototype robot. From the captured EEG signals, P300 and non-P300 are classified using an artificial neural network. In another experiment, we classify signals captured during the intentional blink of the eye and signals where there is no blink. Also, we classify when the user intentionally blinks two, three and four times. From the experiments, we found that $P 300$ cannot be successfully detected with a single dry electrode on Fp1 position. Additionally, we found that signals which contain blink and those which do not contain blink can be classified using an artificial neural network. We also found that different number of blinks can be classified using an artificial neural network. Different number of blinks is used to move forward, turn left and right. The model trained to classify between blink and nonblink signals is used to apply the brake. Experiments performed have shown that using a single-electrode commercial headset and blink of the eye, a user can successfully control the prototype to reach a predefined destination.
\end{abstract}

Keywords: Electroencephalography, machine learning, brain-computer interface, neural networks.

PEOPLE who are impaired from movement due to disabilities or stroke face constant challenges to move around. Event-related desynchronization (ERD)-based wheelchairs depend on the desynchronization in the firing of neurons when a subject imagines movement which was previously in synchrony before imagination. Left hand, right hand and feet imagination by the subject is usually used to control the wheelchair ${ }^{1-4}$. P300-based wheelchairs depend on an odd ball paradigm, where a user is presented with a visual or audio stimulus and he/she is asked to focus on the desired stimulus. When the user focuses on the desired stimulus, a positive deflection in the elec-

*For correspondence. (e-mail: rahulyumcontact@gmail.com) troencephalogram (EEG) occurs with a latency of 250 $500 \mathrm{~ms}$. Usually, the stimulus of forward, left, right is shown to the user randomly and he/she is asked to concentrate on the stimulus of the desired direction. The corresponding stimulus whose signal contains P300 is then used as a command to the wheelchair ${ }^{5-8}$. Steady state visual evoked potential (SSVEP)-based wheelchairs depend on the response of the EEG signal when a visual stimulus is flashed at a particular frequency. A different stimulus is presented to the subject which is flashed at a different frequency. The stimulus which the subject is observing is identified by analysing the frequency of the EEG recorded. The EEG is composed mainly of the frequency in which the visual stimulus is flashing ${ }^{9}$. Hybrid-based wheelchairs combine two or more of the approaches mentioned above for control ${ }^{10,11}$. In a study by Geng et al. ${ }^{1}$, the robot was controlled in two ways - subject control mode and automatic control mode. In the subject control mode, the user needs to imagine left hand, right hand and feet to control the robot while in automatic control mode, the robot is controlled using the sensors installed. In the study by Rebsamen et al. ${ }^{6}$ the robot travelled only on predefined path menus that were shown to the user. Then the user selects the path using P300. Escolano et al. ${ }^{8}$ reported a P300 control robot which can be controlled remotely and interact with the environment, where the user was presented with a set of points generated from the remote environment for navigation, and he/she selected the point using $\mathrm{P} 300$. In the study by Mandel et al. ${ }^{9}$, a route in the environment was selected using SSVEP and an extension of the nearness diagram navigation method was used to navigate the robot to the selected route. P300 and SSVEP can be elicited simultaneously and combining them improves detection of control and idle states ${ }^{10}$. Long et $a l{ }^{11}$ combined P300 and ERD to control a simulated wheelchair. Imageries of left hand and right hand were used to turn left and right. P300 was used to accelerate the wheelchair and imagery of feet was used to decelerate it. EEG signals were acquired mainly using 10-20 electrode placement standards. Rahul et al. ${ }^{12}$ concluded that exploring the option of sleeker design commercial Brain Control Interface (BCI) which uses dry electrode and is more comfortable to wear will be a good research direction. Majority of the research in this field uses multiple electrodes which are costly and not readily available, 

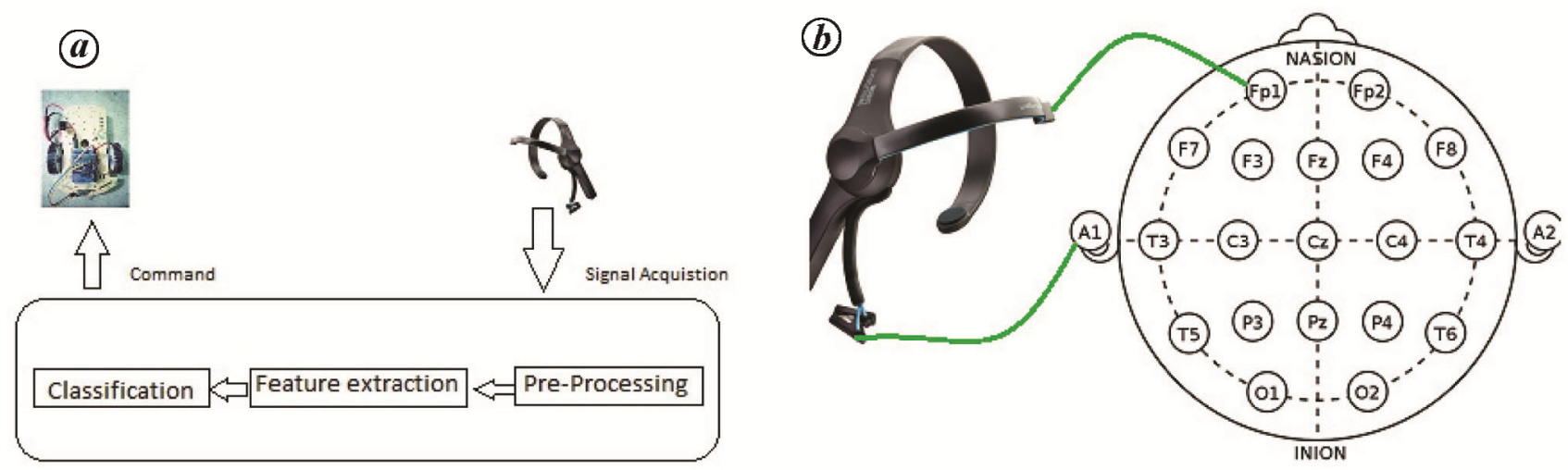

Figure 1. $\boldsymbol{a}$, General System Architecture. $\boldsymbol{b}$, Electrode placement of Neurosky Mindwave Mobile.

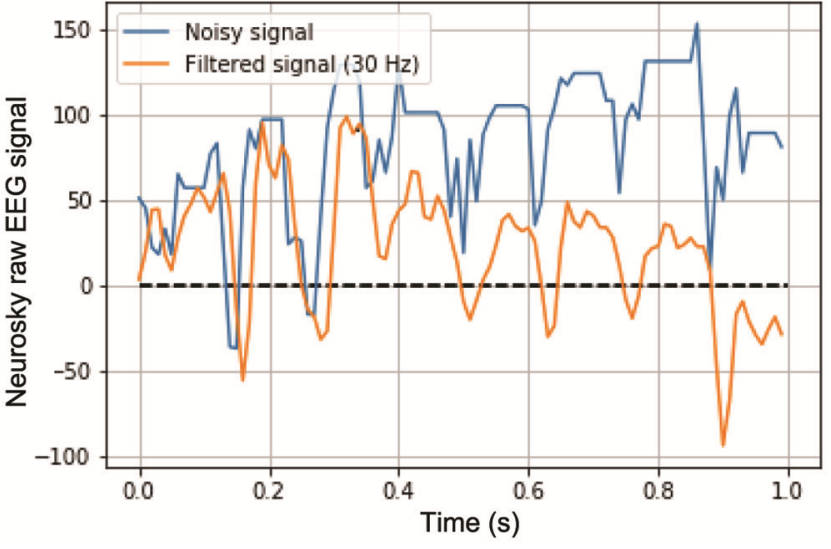

Figure 2. Recorded and filtered signal for P300 detection.

although they give better results. Recent advancements in commercial EEG have seen the use of cheap non-invasive EEG headsets in games. These are comfortable to wear and easily available for sale. They do not need an expert in placing the electrode. This article presents the design and analysis of a mobile prototype robot that can be controlled using a single-electrode commercial EEG headset (Neurosky's MindWave mobile). We look into the possibility of detecting P300 or intentional blink of the eye to control the prototype robot. All the experiments presented in this study are performed with an healthy subject.

\section{System architecture}

First, we acquired signals using a single-electrode EEG headset (Neurosky's MindWave mobile). Next these signals were transferred to a laptop via the headset bluetooth. We then performed pre-processing of the recorded EEG signals and features were extracted from these signals. Next, we performed classification of the signals recorded using the extracted features. The output of the classification algorithm was sent as a command to control the prototype robot via bluetooth. Figure $1 a$ shows the general system architecture.

\section{Methodology}

\section{Signal acquisition and pre-processing}

We performed signal acquisition differently for P300 detection and blink detection; it was done in a room with little distraction. The electrode of the headset was carefully placed at the Fp1 position and the reference electrode at A1 position. Figure $1 b$ shows the electrode placement position. For P300 detection, three arrows pointing forward, left and right were shown to the subject randomly for $1 \mathrm{sec}$ each and he/she was instructed to actively focus on the target direction. EEG data were sampled every $10 \mathrm{~ms}$ for each direction for $1 \mathrm{sec}$ and stored separately. According to Bougrain et al. ${ }^{13}$, the most common filter used for P300 detection is the 0.1$30 \mathrm{~Hz}$ bandpass filter; so the signals recorded were filtered using the same bandpass filter. Figure 2 shows the noisy signal and filtered signal. The experiment was performed for 30 sessions and each session lasted $10 \mathrm{~min}$. From the signals recorded for each direction, those that contained P300 were grouped together, whereas signals that did not contain P300 formed a separate group.

For blink detection, we performed two experiments. Signal acquisition was done differently for each experiment. The first experiment performed classification between signal which contained blink and signal which did not. The second experiment performed classification between different number of blinks. In the first experiment, each trial lasted for $2 \mathrm{sec}$ and signals were sampled every $10 \mathrm{~ms}$. In each trial, the subject was instructed to blink two times or not to blink at all. Thirty trials consisted of one session. The experiment was carried out for eight sessions. From the EEG signal, it was found that blink produces a large positive peak followed by a large negative peak. Also, it has been observed that when the 
user blinks intentionally the amplitude is relatively larger from blinks that occur involuntarily. Figure 3 shows intentional blinking for three times. Figure 4 shows a comparison between involuntary and intentional blinking. In the second experiment, to accommodate different number of blinks, signal recording lasted longer. Each trial lasted for $3.5 \mathrm{~ms}$ and signals were recorded every $10 \mathrm{~ms}$. During each trial, the subject was instructed to perform either two, three or four blinks or no blink. Altogether four sessions containing 30 trials were performed for each type of blink and also for no blink. A sound was played to indicate the start and end of each trial in both the experiments. Once the start sound is played, the user starts blinking. From Figures 4 it is evident that with intentional blinks, large positive and negative deflections occur.

\section{Features extraction and classification}

After signal acquisition, we performed the features extraction and classification of both P300 and blink. For P300 detection, we performed two experiments with

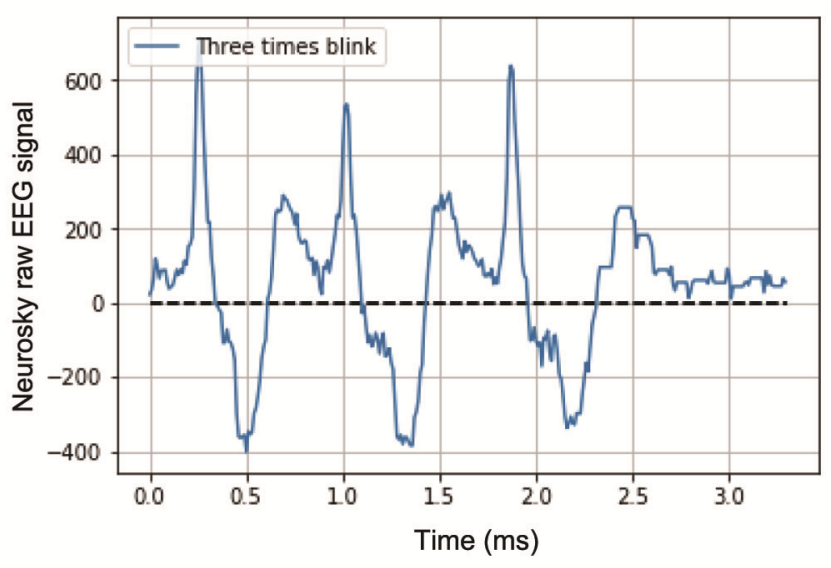

Figure 3. Three times blink signal.

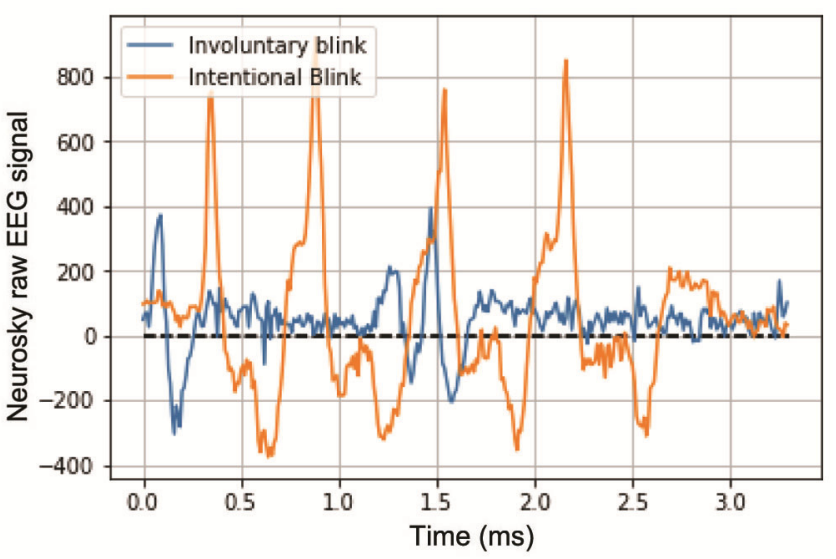

Figure 4. Comparison between Involuntary Blink and Intentional blinking. respect to its features extraction technique. In the first experiment, EEG was again filtered using moving average with a window of 5 from $0-800 \mathrm{~ms}$ to flatten out the signal. To remove any extra noise, the signal was downsampled by a factor of 5 . These values of the EEG signals act as features. Similar features extraction technique has been used by Escolano et al. $^{8}$. In the second experiment, data from 200 to $600 \mathrm{~ms}$ were extracted. If $S$ is the signal between 200 and $600 \mathrm{~ms}$, then the following features were extracted

Positive peak $=S_{\max }$; maximum value of $S$,

Positive peak latency $=t\left(S_{\max }\right)$; time of $S_{\max }$,

Negative peak $=S_{\min }$; minimum value of $S$,

Negative peak latency $=t\left(S_{\min }\right)$; time of $S_{\min }$,

Peak-to-peak $=$ positive peak - negative peak,

Peak-to-peak latency $=t\left(S_{\max }\right)-t\left(S_{\min }\right)$,

$$
\mathrm{RMS}=\sqrt{\frac{1}{n} \sum_{i=1}^{n} S_{1}^{2}} ; \text { root mean square of the signal. }
$$

Positive peak is the maximum value of the EEG signal between $200 \mathrm{~ms}$ to $600 \mathrm{~ms}$. Positive peak latency is the time at which a positive peak occurs. Negative peak is the minimum value of the EEG signal between 200 and $600 \mathrm{~ms}$ and negative peak latency is the time at which a negative peak occurs. Peak-to-peak is the difference between positive and negative peaks. Peak-to-peak latency is the difference between positive peak latency and negative peak latency. These are some of the morphological features specified by $\mathrm{Khemri}^{14}$ for P300 detection. P300 signal is labelled as ' 1 ' and non-P300 signal are labelled as ' 0 '.

For blink detection, we performed two experiments. The first experiment performed classification of two times blink and no blink. The second experiment performed classification of the different number of blinks. In the first experiment, trials which contained blinks and those which did not contain blinks were grouped separately. The first 160 from 200 samples were extracted. In the second experiment, the first 300 from 350 samples were extracted. From these extracted samples, for every 20 samples, we extracted the minimum negative value. If the negative value was not present within the 20 samples, we used 0 as the corresponding extracted value. Also, we calculated the RMS value of the extracted samples. If $S$ is the extracted samples, then

$$
S_{\forall i\{0, \ldots, j\}}=\min \left\{S_{i * 20}, \ldots, S_{((i * 20)+20)}\right\},
$$



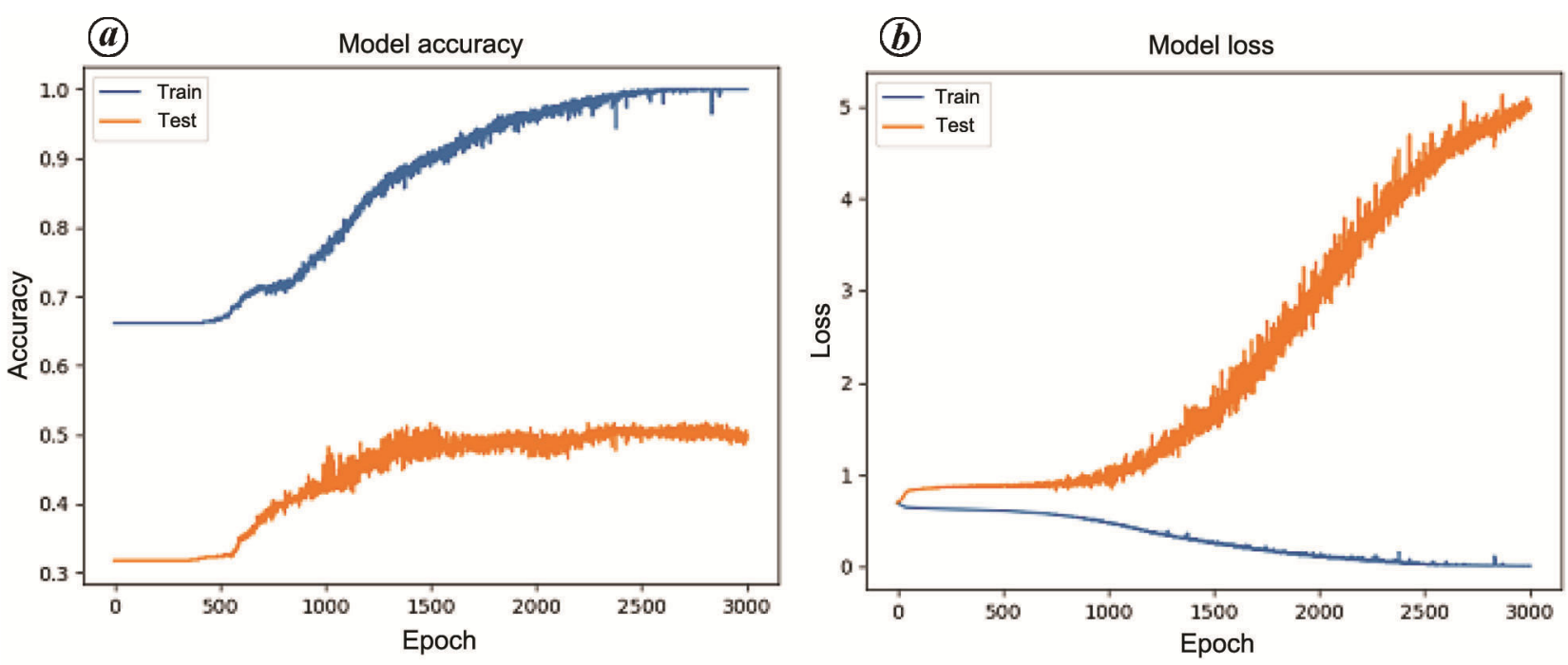

Figure 5. $\boldsymbol{a}, \mathrm{P} 300$ classification-accuracy obtained in experiment $1 . \boldsymbol{b}, \mathrm{P} 300$ classification-loss plot in experiment 1 .
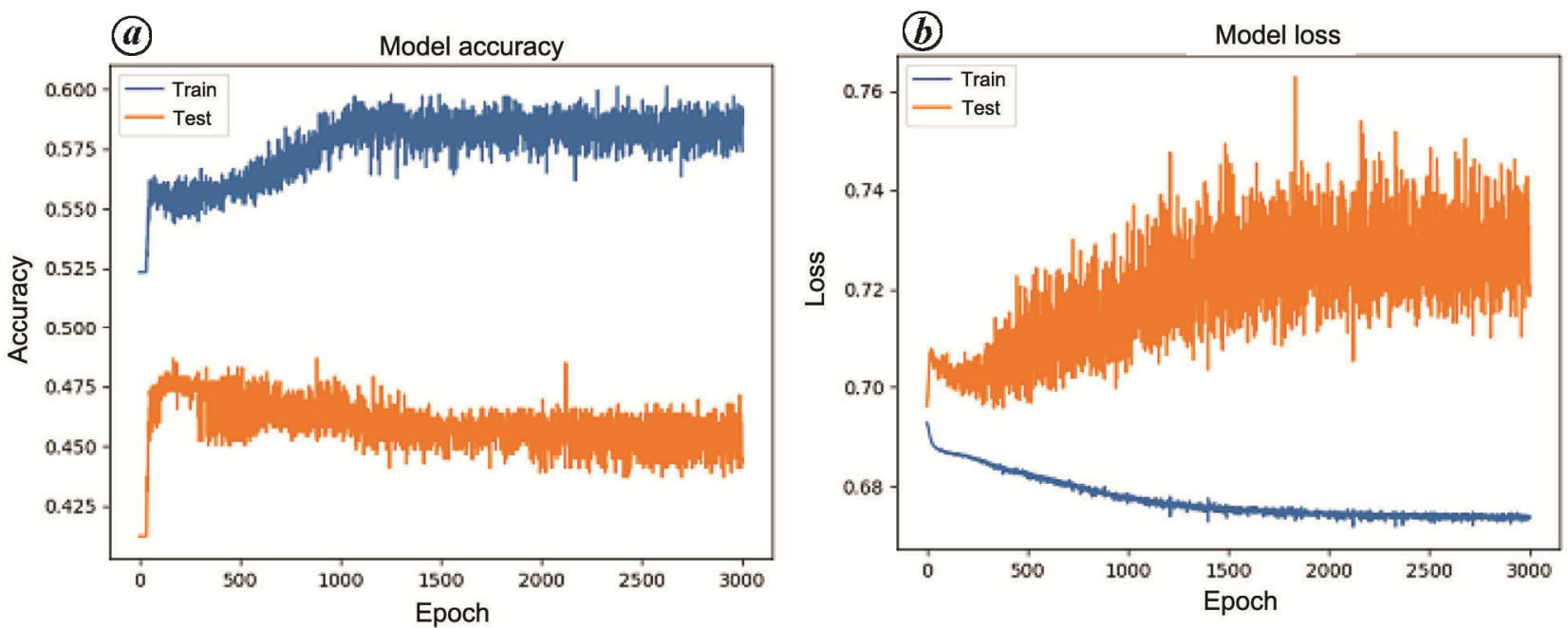

Figure 6. $\boldsymbol{a}, \mathrm{P} 300$ classification- accuracy obtained in experiment 2. $\boldsymbol{b}$, P300 classification-loss obtained in experiment 2.

$$
\begin{aligned}
& X(i)_{\forall i\{0, \ldots, j\}}=\left\{\begin{array}{cc}
S_{i} & S_{i}<0 \\
0 & S_{i} \geq 0,
\end{array}\right. \\
& \mathrm{RMS}=\sqrt{\frac{1}{n} \sum_{i=1}^{n} S_{i}^{2} .}
\end{aligned}
$$

The value of $X$ was concatenated with the corresponding RMS value and used as a feature. The value of $j$ in eqs (8) and (9) is 7 for the first experiment and 14 for the second experiment since the number of samples extracted is 160 and 300 in each of the experiments respectively. In the first experiment, all the trials which contained intentional blinks were labelled as ' 1 ' and those which did not contain blinks were labelled as ' 0 '. In the second experiment, four times blink were labelled as ' 3 ', three times blink as
' 2 ', two times blink as ' 1 ', and signal with no intentional blinks as ' 0 '.

For P300 classification, two multi-layer neural networks with ReLU as activation function for input layer, hidden layers and sigmoid as activation function for output layer were trained separately using back propagation and mini-batch stochastic gradient descent with momentum as an optimizer to classify P300 and non-P300 signals. Features extracted in the first experiment were used for the first network and those extracted in the second experiment were used for the second network in training. The network was trained with the train set and the model was validated with a test set. Figures 5 and 6 show the accuracy and loss of the network in both the experiments. Learning rate $\eta$ and momentum were set to 0.00005 and 0.9 for the first experiment, and 0.006 and 

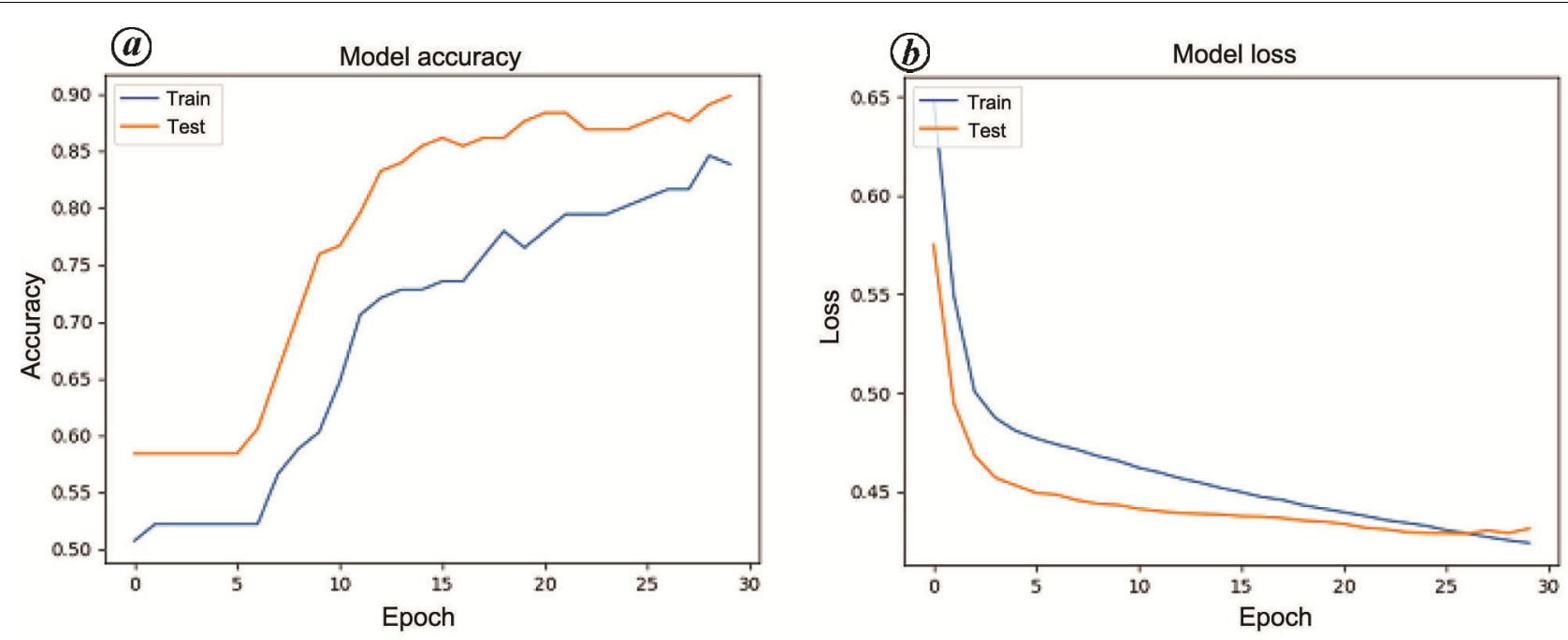

Figure 7. $\boldsymbol{a}$, Accuracy plot obtained in two times blink and non-blink classification. $\boldsymbol{b}$, Loss plot obtained in two times blink and non-blink classification.
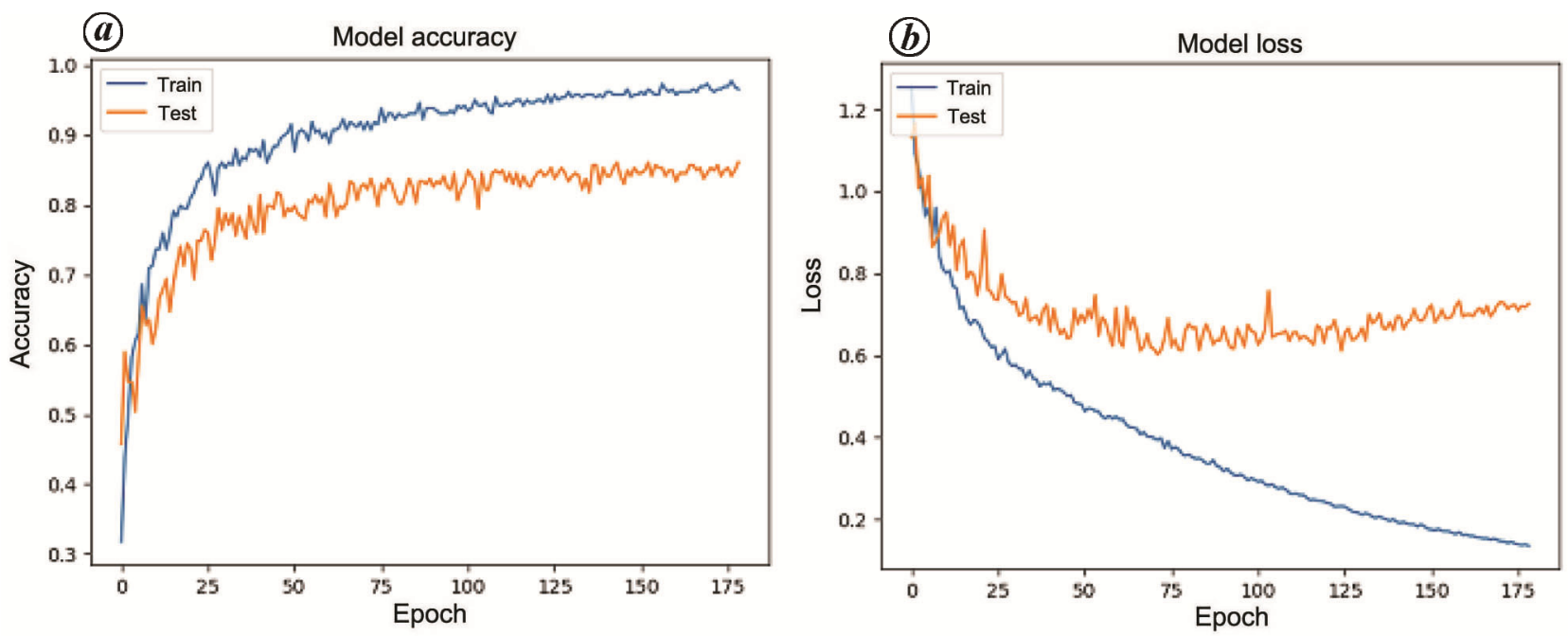

Figure 8. $\boldsymbol{a}$, Accuracy plot obtained in different number of blink classification using Adagrad. $\boldsymbol{b}$, Loss plot obtained in different number of blink classification using Adagrad.

0.2 for the second experiment respectively. Other hyperparameters were set to default value. For blink detection, two multi-layer neural networks with ReLU as activation function for the input layer, hidden layers and sigmoid as activation function for the output layer were trained separately using backpropagation, one for each feature extraction technique. In the first experiment, classification of two times blink and no blink was performed. Optimizer used was Adagrad with the learning rate as 0.0002 . Other hyper-parameters were set to default value. Figure 7 shows the accuracy and loss obtained. In the second experiment, classification of two, three and four times blink was done. Training was performed using stochastic gradient descent with momentum, RMSprop, Adam and Adagrad as optimizers in separate training instances. Learning rate and momentum of stochastic gradient descent were set to 0.006 and 0.4 respectively. The learn- ing rate of RMSprop, Adam and Adagrad were 0.00006, 0.0002 and 0.008 respectively. These learning rates were obtained by performing an exhaustive search from a pre-specified subset of learning rate and momentum. The models were trained using the training set and validated using the test set. Figure 8 shows the accuracy and loss obtained while using Adagrad as optimizer. We presented experiment performed with Adagrad only because it achieves the highest accuracy among other optimizer with which we have performed the experiment.

\section{Controlling prototype robot}

We found that P300 cannot be successfully detected and therefore, used blink to control the robot. The prototype robot consists of an Arduino, two servos, a servo shield 
and a bluetooth module. The servo motor shield is placed on top of the Arduino. Figure 9 shows the components of the robot that are connected to the servo motor shield. The robot is programmed to receive command from the laptop through bluetooth and performs the movement command. To control the direction of the robot, we used trained model to detect two times blink, three times blink, four times blink and no blink. Four times blink is used to move forward, three times and two times to turn left and right respectively. Once the robot moves forward brake mode is activated where the user needs to blink two times in order to apply the brake. Once the brake is applied, it returns to the direction control mode. In Algorithm 1, 'extract feature (S)' extracts the features that will be used for classification. In Algorithm 2, 'model blink (X)' is the trained model to classify the different number of blinks. It predicts whether the EEG data recorded are two, three, four times blink, or no blink. In Algorithm 3 'model

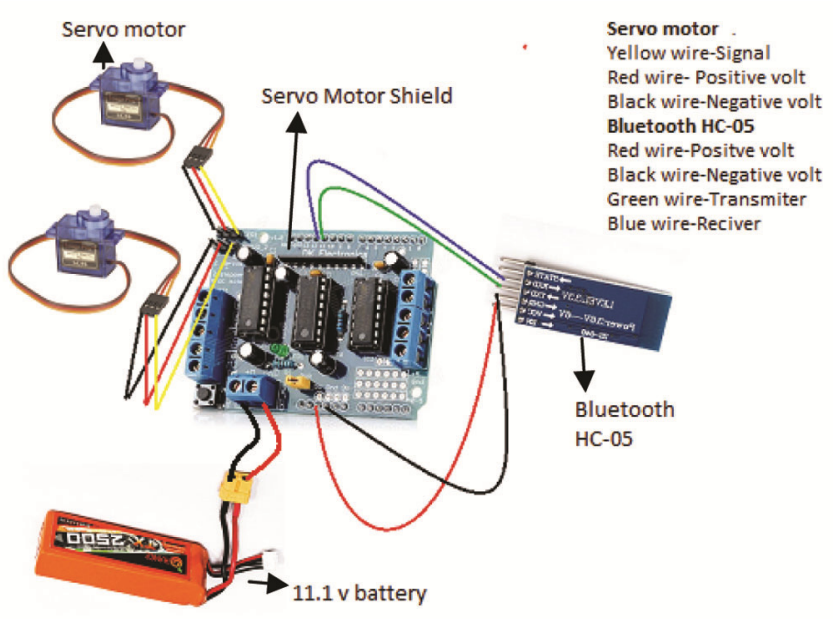

Figure 9. Robot's circuit connection.

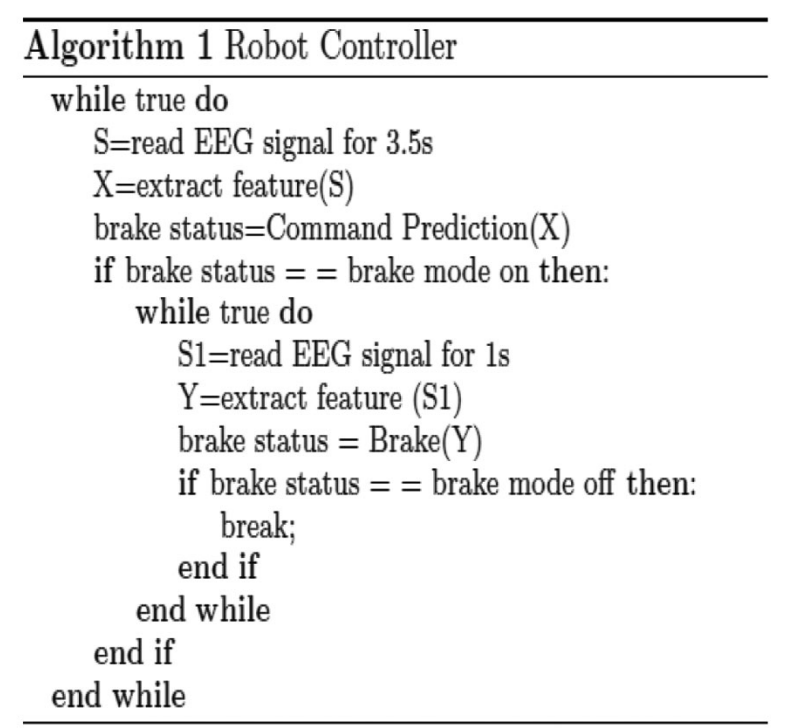

brake (Y)' is the trained model to classify blink and nonblink, which is used to apply the brake.

\section{Experimental analysis and results}

To evaluate how well we can classify P300 and nonP300, blink and non-blink, and different number of blinks, we need to analyse the accuracy obtained. The performance of the robot is analysed with respect to the total command issued, the correct command executed, the wrong command executed, path length, and time taken to cover the path. The subject has to reach a predefined destination following a path of $7.5 \mathrm{~m}$. The path is shown in Figure 10. All the experiments were performed using the dataset obtained in signal acquisition.

For P300 classification Figures 5 and 6 present the accuracy and loss of the network in classifying. For blink detection, Figure 7 shows the accuracy and loss obtained in classifying two times blink and no blink. Accuracy and loss obtained in classifying different number of blinks are shown in Figure 8. In Figures $5 b$ and $6 b$, while classifying P300 and non-P300, the train-set loss keeps decreasing while training until convergence but the test-set loss keeps increasing while testing. This indicates that the network is not able to generalize between training and testing set. A single dry electrode at Fp1 position
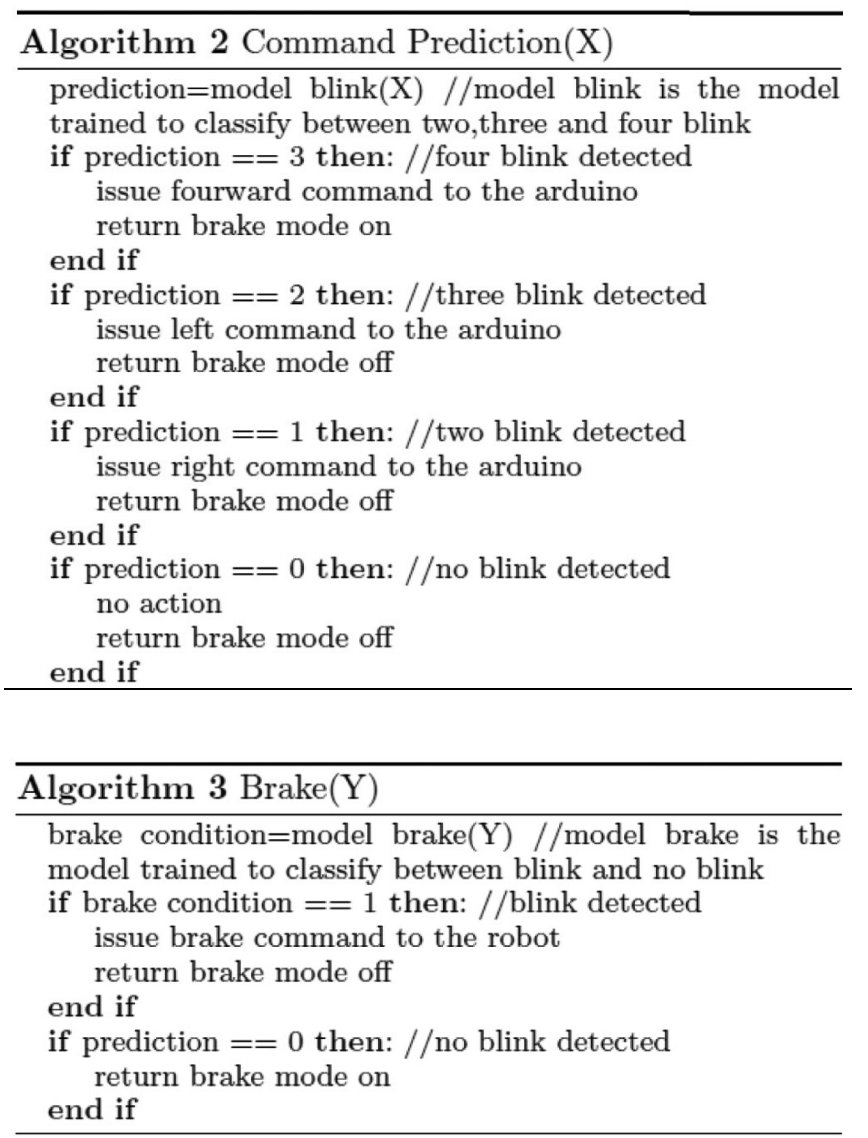


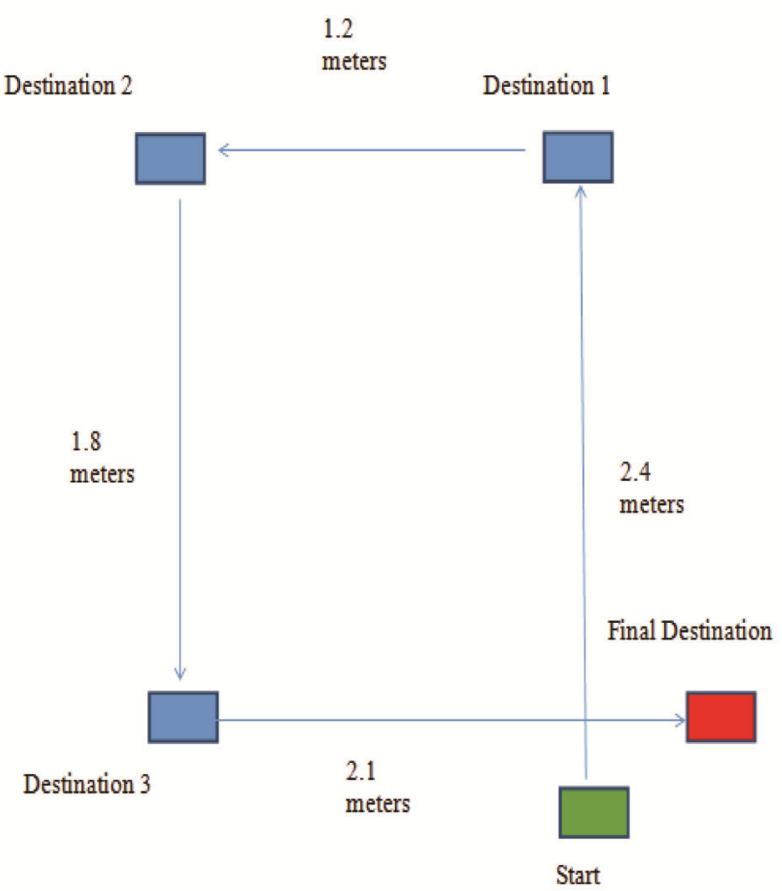

Figure 10. Path used to test the performance of robot.

Table 1. Performance of the robot

\begin{tabular}{lcccc}
\hline Trial no. & Total & Correct & Wrong & Time taken (min) \\
\hline 1 & 21 & 16 & 5 & 7.13 \\
2 & 23 & 16 & 7 & 8.16 \\
3 & 32 & 24 & 8 & 10.43 \\
4 & 22 & 16 & 6 & 7.56 \\
5 & 26 & 21 & 6 & 8.27 \\
\hline
\end{tabular}

detects several blinks and muscular activity. Averaging over the different channels to reduce noise is usually employed for P300 detection, which is not possible here since we are using one electrode. Filtering action also failed to remove noise. This noise produces positive and negative deflections, which lead to a lot of similarity between P300 and non-P300, resulting in overfitting. In both the experiments of classifying P300 and non-P300, we obtained a classification accuracy of just $45-50 \%$. Our experiment of detecting P300 using single dry electrode headset at the Fp1 position was not successful. In the experiment for classifying between two times blink and non-blink, we obtained a classification accuracy of 89-93\%. Loss plot in Figure $7 b$ shows that the test-set loss stops decreasing after some iterations, which indicates that some of the samples cannot be classified properly into blink or non-blink. This may be due to wrong labelling of the samples. In the experiment for classifying the different number of blinks, network trained with stochastic gradient descent, RMSprop, Adam gave classification accuracy of $80-83 \%$. Using Adagrad, classifica- tion accuracy obtained was $84-86 \%$. The slight increase in classification accuracy of Adagrad is due to the sparse nature of the features extracted where Adagrad is known to perform well compared to the other optimization algorithms. In Figure $8 b$ test-set loss increases slightly after some iterations, which indicates this too cannot generalize some of the samples. We were not able to achieve an accuracy of $100 \%$ because of wrongly assuming a trial of a specific number of blinks and labelling it wrongly. Since the process of signal acquisition lasts for several hours, there are chances of error. Galan $e t a l .^{2}$ reported that ERD-based wheelchair subjects need to cope with generating stable EEG patterns to control their wheelchair. Stable EEG pattern generation is not needed in the present system, since the effect of blink on EEG is similar for every person. It will produce positive and negative deflection.

Performance of the robot was analysed using five trials in which the subject had to reach the predefined destination. It was found that subject could reach the destination in all of the trials. Table 1 gives the performance of the robot in each trial.

\section{Discussion and conclusion}

P300 is hard to detect using Neurosky MindWave since it contains a lot of noise from blink. It may be due to the position of the electrode of MindWave mobile headset, which was fixed at the Fp1 position. We conclude that blink can be used as an alternative to control the wheelchair. It is relatively easy and cheap to do so. The experiment was performed with healthy subject. Verification with a non-healthy subject is still needed. Most researchers in this field utilize signal acquisition system which is meant for laboratory use. Moreover, wet electrodes are mainly used. Exploring the option of teaching the robot about obstacle avoidance or path planning will be a good research direction.

1. Geng, T., Gan, J. Q. and Hu, H., A self-paced online BCI for mobile robot control. Int. J. Adv. Mech. Syst., 2010, 2(1-2), 2835.

2. Galán, F., Nuttin, M., Lew, E., Ferrez, P. W., Vanacker, G., Philips, J. and Millán, J. D., A brain-actuated wheelchair: asynchronous and non-invasive brain-computer interfaces for continuous control of robots. Clin. Neurophysiol., 2008, 119(9), 2159-2169.

3. Li, J., Liang, J., Zhao, Q., Li, J., Hong, K. and Zhang, L., Design of assistive wheelchair system directly steered by human thoughts. Int. J. Neural Syst., 2013, 23(3), 1350013.

4. Barbosa, A. O., Achanccaray, D. R. and Meggiolaro, M. A., Activation of a mobile robot through a brain computer interface. In IEEE International Conference on Robotics and Automation, Alaska, USA, 3 May 2010, pp. 4815-4821.

5. Pires, G., Castelo-Branco, M. and Nunes, U., Visual P300-based BCI to steer a wheelchair: a Bayesian approach. In 30th Annual International Conference of the IEEE Engineering in Medicine and Biology Society, Vancouver, Canada, 20 August 2008, pp. 658661. 


\section{RESEARCH ARTICLES}

6. Rebsamen, B., Guan, C., Zhang, H., Wang, C., Teo, C., Ang, M H. and Burdet, E., A brain controlled wheelchair to navigate in familiar environments. IEEE Trans. Neural Syst. Rehab. Eng., 2010, 18(6), 590-598.

7. Shin, B. G., Kim, T. and Jo, S., Non-invasive brain signal interface for a wheelchair navigation. In International Conference on Control, Automation and Systems, 27 October 2010, pp. 2257 2260 .

8. Escolano, C., Murguialday, A. R., Matuz, T., Birbaumer, N. and Minguez, J., A telepresence robotic system operated with a P300based brain-computer interface: initial tests with ALS patients. In 2010 Annual International Conference of the IEEE Engineering in Medicine and Biology, Buenos Aires, Argentina, 31 August 2010, pp. 4476-4480

9. Mandel, C., Lüth, T., Laue, T., Röfer, T., Gräser, A. and KriegBrückner, B., Navigating a smart wheelchair with a braincomputer interface interpreting steady-state visual evoked potentials. In IEEE/RSJ International Conference on Intelligent Robots and Systems, St Louis, USA, 10 October 2009, pp. 1118-1125.

10. Li, Y., Pan, J., Wang, F. and Yu, Z., A hybrid BCI system combining P300 and SSVEP and its application to wheelchair control. IEEE Trans. Biomed. Eng., 2013, 60(11), 3156-3166.
11. Long, J., Li, Y., Wang, H., Yu, T., Pan, J. and Li, F., A hybrid brain-computer interface to control the direction and speed of a simulated or real wheelchair. IEEE Trans. Neural Syst. Rehab. Eng., 2012, 20(5), 720-729.

12. Rahul, Y., Sharma, R. K. and Nissi, P., A review on EEG control smart wheel chair. Int. J. Adv. Res. Comput. Sci., 2017, 8(9), 501507.

13. Bougrain, L., Saavedra, C. and Ranta, R., Finally, what is the best filter for P300 detection? In TOBI Workshop 11l-Tools for BrainComputer Interaction, Würzburg, Germany, 20 March 2012.

14. Khemri, N. A., P300 wave detection using a commercial noninvasive EEG sensor: reliability and performance in control applications. Masters Abst. Int., 2012, 51(2).

Received 17 September 2018; accepted 12 March 2019

doi: $10.18520 / \mathrm{cs} / \mathrm{v} 116 / \mathrm{i} 12 / 1993-2000$ 